\title{
Understanding complex MHD activities associated with a relaxation in HT-7 tokamak
}

Erzhong $\mathrm{Li}^{1}$, Liqun $\mathrm{Hu}^{1}$, V. Igochine ${ }^{2}$, O. Dumbrajs ${ }^{3}$, and Kaiyun Chen ${ }^{1}$

1 Institute of Plasma Physics, Chinese Academy of Science, Hefei 230031, China

2 MPI fur Plasmaphysik, Euratom-Association, D-85748 Garching, Germany

3 Institute of Solid State Physics, Association Euratom-University of Latvia, Kengaraga Street 8, LV-1063, Riga, Latvia

$$
\text { E-mail: lqhu@ipp.ac.cn (Liqun Hu) }
$$

\section{Abstract}

A new relaxation instability with complex magnetohydrodynamics (MHD) activities has been found in the HT-7 tokamak operational region, which manifests itself in the bursts of hydrogen alpha-ray radiations, electron cyclotron emission (ECE), and soft $\mathrm{x}$-ray (SX) radiations on outer channels, as well as the complex MHD perturbations, but without hard disruptions. The MHD modes were indentified as $\mathrm{m} / \mathrm{n}=2 / 1, \mathrm{~m} / \mathrm{n}=3 / 2$, and $\mathrm{m} / \mathrm{n}=5 / 3$ just before the relaxation ( $\mathrm{m}$ and $\mathrm{n}$ are poloidal and toroidal mode number, respectively). The Poincaré mapping, according to the measured perturbations of the MHD modes at edge, was used to show the topology of magnetic field lines. It was found that a stochastic annular belt was resulted just before the relaxation due to the $m / n=5 / 3$ island 
overlapping with $\mathrm{m} / \mathrm{n}=2 / 1$ and $\mathrm{m} / \mathrm{n}=3 / 2$ islands. That qualitatively coincided with the results of SX tomography.

\section{Introduction}

Macroscopic magneto-hydro-dynamics (MHD) instability plays an important role from the viewpoint of maximum achievable plasma parameters associated with tokamak safety operation [1]. The low q (q is safety factor) limit and/or density limit disruptions are usually observed with complicated precursor oscillations [2]. Probably, the nonlinear interaction of MHD modes eventually leads to disruptions [3]. The resulting runaway electrons and heat power could damage the first wall [4]. Hence, the study of MHD instabilities is important in the field of magnetic confinement plasma.

A long-term problem is the core periodic relaxation, known as sawtooth instability, which was first observed in ST tokamak [5-6]. Then the Kadomtsev full magnetic reconnection model was used to explain the sawtooth in ST [7]. However, that model cannot account for the fast sawtooth crash in JET [8]. In addition, some novel sawtooth, observed as compound, giant and monster sawtooth, also challenged the Kadomtsev model [9-11]. There is hitherto no unified model which could explain this kind of core instability in different machines [12-13]. Even if in the same machine, different heating methods probably bring in different effects on magnetic field reconnection, manifesting different phenomena on 
sawtooth [13-19].

The coexistence of tearing modes with different helicity further complicated not only the sawtooth, but also the disruption instability [20-24]. The mechanisms of disruptions have not been well understood from the view point of MHD instability up to the present. Two categories of explanations seemed reasonable [25]. One was the single helicity model which claimed that a large $\mathrm{m} / \mathrm{n}=2 / 1$ island interacted with external perturbation field (e.g. mode locking). The other proposed that the interaction among tearing modes of different helicity caused the stochastization of magnetic field lines leading to disruptions. It should be mentioned that, in recent years, the stochastization has also been suggested as a possible explanation for the fast sawtooth crash in ASDEX-Upgrade tokamak [26-27].

In present HT-7 experiments, a large number of discharges show the behaviors of relaxation which differ from the usual sawtooth crash as introduced above, and which are also not regarded as hard disruptions due to mode locking because the discharges could recover after a relaxation. Each relaxation is embodied in rapid enhancement of the hydrogen alpha-ray $\left(H_{\alpha}\right)$ radiations, electron cyclotron emission (ECE), and soft $\mathrm{X}$-ray (SX) radiations on outer channels, as well as the bursts of magnetic perturbations (Mirnov signals). The fast Fourier transform (FFT) analysis of Mirnov signals shows a broaden spectrum in addition to several 
coherent peaks which motivate us to understand the complex MHD mode structures.

Following section describes the diagnostics and experiments. In section 3, Experimental observations are shown. Comparison between experiments and numerical calculations is presented in section 4. Finally, we summarize the main results in section 5 .

\section{Descriptions of diagnostics and experiments}

HT-7 is a medium-sized tokamak with circular section, of which the major radius is $R_{0}=1.22 \mathrm{~m}$ and minor radius $a=0.27 \mathrm{~m}$ [28]. The main diagnostics used here are briefly introduced. A 16-channels heterodyne radiometer is used for electron cyclotron emission (ECE) which covers frequency region $98-126 \mathrm{GHz}$ [29]. Three soft X-ray (SX) arrays are employed for tomography analysis. They are located respectively on horizontal, up-vertical and down-vertical windows in one poloidal cross section, and each array has 46 chords covering the whole plasma region [15]. The characteristics of MHD modes could be identified by magnetic probes, consisting of a poloidal and a toroidal Mirnov-coils array fixed on the inside of vacuum vessel. The poloidal array has 12 probes, while the toroidal one has 9 probes. The confinement properties were monitored by a 35-channels $H_{\alpha}$ photodiode array. The diagnostics involved in our experiments are schematically shown in figure 1 , where the $H_{\alpha}$ chord passes through the plasma near to center. The two SX chords in the SXC 
array are prominently drawn which are close to two sampling locations of the ECE diagnostic.

Pure ohmic discharges were performed to exclude auxiliary heating effects (fast particles effect or current driving effect). Figure 2 shows a waveform of the typical shot 110225 . The plasma current is about $120 \mathrm{kA}$. The edge safety factor is $q_{a}=6.6$. The central safety factor is found $q_{0}>1$ because of absence of $\mathrm{q}=1$ rational surface on $\mathrm{SX}$ signals. The line-averaged density is about $n_{e} \approx 3.5 \times 10^{19} \mathrm{~m}^{-3}$. Along with MHD bursts as observed on Mirnov signals, SX signals crash at $\mathrm{r}=-2.44 \mathrm{~cm}$, whereas the positive spikes appear on $H_{\alpha}$ signals, on ECE signals at $\mathrm{r}=-15.2 \mathrm{~cm}$ and on SX signals at $\mathrm{r}=-15.8 \mathrm{~cm}$. Meanwhile, the plasma current drops near $3 \%$. It seems puzzled, as observed in figure 2, that the SX signals at $\mathrm{r}=-2.44 \mathrm{~cm}$ show a crash while the ECE signals at $\mathrm{r}=2.6 \mathrm{~cm}$ have a positive spike. However, one should note that the SX radiations reflect the plasma pressure [15]. Moreover, the ECE emission is a measure of electron temperature. Thus, this phenomenon (decreasing SX and increasing ECE) probably implies the deterioration of particle confinement. A detailed discussion about that is beyond the scope of this paper.

A series of discharges were performed to investigate the dependence of this kind of instability on the major plasma parameters. The results are shown in operational region (figure 3). Relaxations with complex MHD activities (solid circles) occur with plasma current $I_{p}=80-130 \mathrm{kA}$, central 
line averaged electron density $n_{e}=2-4 \times 10^{19} \mathrm{~m}^{-3}$ and central electron temperature $\sim 1 \mathrm{keV}$. The solid line is a linear fit to the data denoted by solid circles. The normal discharges without MHD activity were denoted by open circles, while the quadrangle surface ' $\mathrm{D}$ ' indicates disruptions observed in previous HT-7 experiments [30-31].

\section{Experimental observations of MHD modes}

As indicated above, it is the purpose of this paper to understand the complex MHD activities during the phase of relaxation, and thus to peer at the magnetic field topology. Figure 4 shows the magnetic perturbations (figure 4(a)) and its time frequency spectrum (figure 4(b)) during a relaxation. The arrows indicate the main MHD modes identified from Mirnov signals by fast Fourier transform (FFT) method combined with singular value decomposition (SVD) analysis, which could identify the MHD modes at different frequency bands [32]. It is observed that the mode $\mathrm{m} / \mathrm{n}=3 / 2$ is firstly destabilized at about $\mathrm{t}=438.2 \mathrm{~ms}$, and then the mode $\mathrm{m} / \mathrm{n}=5 / 3$ is stirred. The broad spectrum $\left(f_{m, n}=30 \sim 50 \mathrm{kHz}\right)$ is also observed, whereas it is difficult to discern their mode numbers from Mirnov signals. It is thus defined as the 'high ( $\mathrm{m}, \mathrm{n})$ modes'. The other notable observation is that the three low-order modes are phase locked $\left(f_{3,2} \approx 2 f_{2,1} ; f_{5,3} \approx 3 f_{2,1}\right)$ in figure $4(\mathrm{~b})$.

Figure 5 shows the power spectrum density of the raw Mirnov signals in figure 4(a). The three coherent peaks are corresponding to the three 
low order modes. The broad spectrum is the characterization of the "high $(\mathrm{m}, \mathrm{n})$ modes'. The dynamic characteristic of the low order modes could be extracted by the FFT filtering method owing to their different frequencies. The results are illustrated in figure 6. It is observed that the mode $m / n=2 / 1$ is saturated just before the excitation of $m / n=3 / 2$ by the comparison between figure 6(b) with figure 6(c). However, the mode $\mathrm{m} / \mathrm{n}=2 / 1$ is further destabilized accompanied by destabilization of the other two modes. It is difficult to discern which one or both of the modes $\mathrm{m} / \mathrm{n}=3 / 2$ in figure $6(\mathrm{c})$ and $\mathrm{m} / \mathrm{n}=5 / 3$ in figure $6(\mathrm{~d})$ further destabilize the mode of $m / n=2 / 1$. It is also hard to distinguish that whether the further growth of $m / n=2 / 1$ mode or the growing $m / n=3 / 2$ mode destabilizes the mode of $m / n=5 / 3$ while the three modes coexist. However, it could be inferred that the interaction among the MHD modes leads to the relaxation near to $\mathrm{t}=439.9 \mathrm{~ms}$ in figure 2 .

The complex MHD activities could also be found in SX radiations as illustrated in figures 6(e) and 6(f). The MHD properties at $\mathrm{t}=438.8 \mathrm{~ms}$ are obviously different from those at $\mathrm{t}=437.5 \mathrm{~ms}$. Note that the mode $\mathrm{m} / \mathrm{n}=3 / 2$ has been destabilized at $\mathrm{t}=438.8 \mathrm{~ms}$ as indicated in figure $6(\mathrm{c})$. In contrast, only the mode $\mathrm{m} / \mathrm{n}=2 / 1$ is present at $\mathrm{t}=437.5 \mathrm{~ms}$. The detailed comparisons can be found in SX tomography plots (figure 7) where the Fourier-Bessel inversion method was applied [15]. At $\mathrm{t}=437.5 \mathrm{~ms}$, only the $\mathrm{m} / \mathrm{n}=2 / 1$ island structure is observed in figure 7(a) where the dash line circle is the 
estimated location of the $\mathrm{q}=2$ rational surface ( $\mathrm{q}$ is the safety factor), while the arrow in figure $7(\mathrm{~b})(\mathrm{t}=438.8 \mathrm{~ms})$ indicates a weaker structure due to the destabilization of $m / n=3 / 2$ mode as shown in figure $6(\mathrm{c})$. At $\mathrm{t}=439.5 \mathrm{~ms}$, the tomography plot in figure 7 (c) manifests the properties of the $m / n=2 / 1$ island due to the large perturbations of $m / n=2 / 1$ island, but with a much weaker structure than that in figure 7(b) because of further growth of the mode $m / n=3 / 2$. It should be noted that the modes $m / n=2 / 1$, $\mathrm{m} / \mathrm{n}=3 / 2$ and $\mathrm{m} / \mathrm{n}=5 / 3$ are simultaneously observed at $\mathrm{t}=439.86 \mathrm{~ms}$, $\mathrm{t}=439.9 \mathrm{~ms}$ and $\mathrm{t}=440 \mathrm{~ms}$. These time points have been marked by vertical arrows in figure 6(f). By comparing figure 7(d) with figure 7(e), one could find a much more constrictive hot core in figure $7(\mathrm{e})(\mathrm{t}=439.9 \mathrm{~ms})$ than that in figure $7(\mathrm{~d})(\mathrm{t}=439.86 \mathrm{~ms})$. That is probably caused by the fast parallel-field transports due to the stochastization of magnetic field lines [15, 33-34]. At $t=440 \mathrm{~ms}$ (just after relaxation), the coherent structure, possessing characteristics of the $\mathrm{m} / \mathrm{n}=2 / 1$ island, still exists, as indicated by the double arrow in figure 7(f).

\section{MHD mode structures based on magnetic field line mapping}

The mapping technique of magnetic field lines based on Hamilton-Jacobi method has been discussed specifically, and applied in ASDEX-Upgrade [35-38]. It is implemented in our experiments to understand the topology of magnetic field lines with the observed magnetic islands, because the above experimental analysis has proposed that the stochastic magnetic 
field lines were probably responsible for the relaxation. This section aims to clarify which islands in SX tomography plots could lead to the stochastization of magnetic field lines. Based on those, it is necessary to obtain Poincaré mapping. The mapping technique requires the knowledge of perturbation Hamiltonian $H_{m n}$ and safety factor profile (q-profile). The determination of both parameters is a challenging task in experiments.

As mentioned in previous sections, we only have two points (the resonance surface of $\mathrm{q}=2$ and the edge value) to determine the q-profile. Thus, it could be parameterized as follows:

$$
q(\rho)=C_{0}+\lambda \rho^{2}
$$

Where, $\rho$ is the radius normalized to the minor radius of plasma (i.e. $\rho=r / a$ ). The coefficients in Eq.(1) are found as $\lambda=5.2$ and $C_{0}=1.4$ according to the position of the rational surface $\mathrm{q}=2$ and the edge safety factor $q_{a}=6.6$. This parameterized q-profile could correctly describe the MHD mode positions in our experiments. As observed in figure 8, the rational surfaces $\mathrm{q}=3 / 2$ and $\mathrm{q}=5 / 3$ are both coved by Eq. (1), which is necessary because the modes $m / n=3 / 2$ and $m / n=5 / 3$ have also been observed besides the mode $\mathrm{m} / \mathrm{n}=2 / 1$ in experiments.

The other parameter for the magnetic field mapping is the $H_{m n}$, which is the normalization of the perturbation flux to the toroidal magnetic flux, and results from the perturbation of MHD modes. Theoretically, it could be obtained by numerically solving the tearing mode equation. However, 
the experimental uncertainties of q-profiles limit the accuracy of numerical solution. The parameterized solution of perturbation flux was already obtained, and confirmed as a rational approximation to the solutions of the tearing mode equation [39]. In Refs.[37-39], it has be parameterized as:

$$
\begin{aligned}
& H_{m n}=\rho_{m n} \alpha\left(\frac{r}{r_{m n}}\right)^{m}\left(1-\beta \frac{r}{r_{m n}}\right) \text { for } r \leq r_{m n}, \\
& H_{m n}=\rho_{m n} \frac{\alpha(1-\beta)-\gamma+\gamma \frac{r}{r_{m n}}}{\left(\frac{r}{r_{m n}}\right)^{m+1}} \quad \text { for } r>r_{m n}
\end{aligned}
$$

to calculate the Poincare mapping. Where, the parameters $\alpha, \beta$ and $\gamma$ are the free parameters to describe the shape of perturbation flux. The parameter $r_{m n}$ is the location of rational surface normalized to the minor radius. The parameter $\rho_{m n}$ is the normalized coefficients by requiring the perturbation flux amplitudes coincident with the experimental measurements $H_{m n}=H_{m n}^{\exp } \equiv B_{m n} / B$ at edge. In this study, the free parameters $\alpha, \beta$ and $\gamma$ are firstly chosen to make sure that they could describe the modes just before the relaxation, and then the same parameters are used in calculations of Poincaré mapping according to the measurement values of magnetic perturbations from Mirnov signals at the other time points. They are ascertained by comparisons with the SX tomography plots. The fitting parameters for each mode are eventually determined in our experiments and inserted in figure 9. It is obvious that 
the parameterized solutions do not lose the general behaviors of tearing modes (e.g. the perturbation flux connects, but the derivation of its logarithm jumps at the resonant surface).

With the experimental measurement values $H_{m n}^{\exp }$ extracted from the Mirnov signals at $\mathrm{t}=439.86 \mathrm{~ms}$, as marked in figure 9 , one can obtain the Poincaré mapping of the magnetic field lines which is illustrated in figure 10. It is found that a stochastic annular belt locates in the mapping of magnetic field lines. Outside of the annular belt, the magnetic field lines are intact. This is due to the fact that, at $\mathrm{t}=439.86 \mathrm{~ms}$, the modes $\mathrm{m} / \mathrm{n}=3 / 2$ and $\mathrm{m} / \mathrm{n}=5 / 3$ coexists with the mode $\mathrm{m} / \mathrm{n}=2 / 1$, and that the adjacent rational surfaces are close to each other leading to overlap of islands. Note that the rational surface of $\mathrm{q}=2$ is estimated as $r_{21} / a=0.34$ from $\mathrm{SX}$ tomography, and that the mode $\mathrm{m} / \mathrm{n}=5 / 3$ locates between $\mathrm{q}=2$ and $\mathrm{q}=3 / 2$ surfaces. Thus, the distance between two adjacent rational surfaces is small. Variations of location of $\mathrm{q}=2$ within experimental errors do not change the overall Poincaré mapping. It means that the Poincaré mapping almost shows the same stochastic annular belt as that in figure 10 .

The same values of parameters $\alpha, \beta$ and $\gamma$ are used to obtain the Poincaré mapping, but with measured perturbations $H_{m n}^{\exp }$ at the other time points. At $\mathrm{t}=437.5 \mathrm{~ms}$, there is only the $\mathrm{m} / \mathrm{n}=2 / 1$ perturbation with the amplitude $H_{21}^{\text {exp }}=4 \times 10^{-4}$. The Poincaré mapping is shown in figure 11 (a) where no stochastic region can be observed. From the Mirnov signals, we 
have known that the $m / n=2 / 1$ was saturated. The SX tomography only showed the $\mathrm{m} / \mathrm{n}=2 / 1$ perturbations. At $\mathrm{t}=438.8 \mathrm{~ms}$, the mode $\mathrm{m} / \mathrm{n}=3 / 2$ has also been destabilized as observed in the Mirnov signals. Its perturbation amplitude is small $H_{32}^{\text {exp }}=8 \times 10^{-6}$, and the perturbation of the mode $\mathrm{m} / \mathrm{n}=2 / 1$ has the same amplitude as that at $\mathrm{t}=437.5 \mathrm{~ms}$. The Poincaré mapping with these perturbations is illustrated in figure 11 (b). It is observed that the $m / n=3 / 2$ island takes shape. However, the width of $\mathrm{m} / \mathrm{n}=3 / 2$ island is small, which does not overlap with the $\mathrm{m} / \mathrm{n}=2 / 1$ island. Thus, the stochastic region could not be observed. At the following time point $(\mathrm{t}=439.5 \mathrm{~ms})$, the perturbation amplitude of the mode $\mathrm{m} / \mathrm{n}=3 / 2$ is $H_{32}^{\text {exp }}=1 \times 10^{-5}$, and that of the mode $\mathrm{m} / \mathrm{n}=2 / 1$ is still near $H_{21}^{\text {exp }}=4 \times 10^{-4}$. The $\mathrm{m} / \mathrm{n}=3 / 2$ island could be conspicuously observed in figure $11(\mathrm{c})$. The two islands of $m / n=3 / 2$ and $m / n=2 / 1$ are still separated. However, the presence of $m / n=3 / 2$ island results in the weak structure in the SX tomography plots (figures 7(b) and 7(c)).

The remnant time point for analysis is $t=440 \mathrm{~ms}$ just after the relaxation, when the three modes of $\mathrm{m} / \mathrm{n}=2 / 1, \mathrm{~m} / \mathrm{n}=3 / 2$ and $\mathrm{m} / \mathrm{n}=5 / 3$ coexist with the measured perturbation values of $H_{21}^{\exp }=7.2 \times 10^{-4}$, $H_{32}^{\text {exp }}=8 \times 10^{-5}$ and $H_{53}^{\text {exp }}=6 \times 10^{-6}$ from Mirnov signals. The Poincaré mapping is shown in figure 11 (d) where a coherent structure, possessing the properties of $m / n=2 / 1$ island as indicated by the double arrow, is still observed even though a large stochastic region exists. This is consistent 
with the SX tomography plot in figure 7(f), qualitatively at least.

\section{Summary}

Discharges with complex MHD activities have been found in the HT-7 tokamak operational region, and have been defined as relaxation because of the behaviors of short bursts on $H_{\alpha}$, ECE, SX (outer channels) and Mirnov signals. It is different from the usual sawtooth because the plasma current drop near 3\%. However, the discharges could recover after relaxation. They are thus ascribed to the category of minor disruption.

Experimental data analysis from Mirnov and SX signals indicated that the saturated $m / n=2 / 1$ mode further grew with the destabilization of the $\mathrm{m} / \mathrm{n}=3 / 2$ mode just before relaxation. The destabilization of the mode $\mathrm{m} / \mathrm{n}=5 / 3$ made the adjacent islands overlapping. The Poincaré mapping, based on the Hamilton-Jacobi method, revealed that the magnetic field lines were stochastic inside an annular belt. Thus, this kind of minor disruption was understood as a stochastic relaxation.

In conclusion, the complex MHD activity resulted from the interaction of the $\mathrm{m} / \mathrm{n}=2 / 1$ mode with $\mathrm{m} / \mathrm{n}=3 / 2$ and $\mathrm{m} / \mathrm{n}=5 / 3$ modes leading to a stochastic annular belt of magnetic field lines, which was responsible for the relaxation in our experiments.

\section{Acknowledgements}

The authors would like to acknowledge all the contributors in HT-7 experiments. Special thankfulness is given to diagnostic team for their 
supports. One of the authors, E. Li, wants to thank Dr. Q. Yu and Prof. H. Zohm (Max-Planck-Institut (MPI) in Germany) for detailed discussions about soft x-ray tomography asymmetry and stochastization respectively. This work is supported by the National Natural Science Foundation of China under Grant No. 10935004.

\section{References}

[1] Hender T C et al 2007 Nucl. Fusion 47 S128

[2] Wesson J A et al 1989 Nucl. Fusion 29641

[3] Schuller F C 1995 Plasma Phys. Control. Fusion 37 A135

[4] Janos A C 1990 J. Nucl. Mater. 176-177 773

[5] von Goeler S et al 1974 Phys. Rev. Lett. 331201

[6] Rosenbluth M N et al 1973 Phys. Fluids 161894

[7] Kadomtsev B B 1976 Sov. J. Plasma Phys. 1389

[8] Wesson J A 1990 Nucl. Fusion 302545

[9] Campbell D J et al 1986 Nucl. Fusion 23147

[10] Pfeiffer W 1985 Nucl. Fusion 25673

[11] Campbell D J 1988 Phys. Rev. Lett. 602148

[12] Aydemir A Y 1990 Phys. Fluids B 22135

[13] Chen K et al 2009 Phys. Lett. A 3724469

[14] Sun Y et al 2005 Plasma Phys. Control. Fusion 47745

[15] Sun Y et al 2009 Plasma Phys. Control. Fusion 51065001 
[16] Li E et al 2010 Chin. Phys. B 19035203

[17] Porcelli F 1991 Plasma Phys. Control. Fusion 331601

[18] Porcerlli F et al 1996 Plasma Phys. Control. Fusion 382163

[19] Bateman G et al 2006 Phys. Plasmas 13072505

[20] Xu X et al 2010 Plasma Phys. Control. Fusion 52015008

[21] Suttrop W et al 1997 Nucl. Fusion 37119

[22] Liang Y et al 2007 Nucl. Fusion 47 L21

[23] Nave M F F et al 1992 Nucl. Fusion 32825

[24] Waidmann G et al 1992 Nucl. Fusion 32645

[25] Howling A A and Robinson D C 1988 Plasma Phys. Control. Fusion 301863

[26] Igochine V et al 2007 Nucl. Fusion 4723

[27] Igochine V et al 2008 Nucl. Fusion 48062001

[28] Wan Baonian 2003 J. Nucl. Mater. 313-316 127

[29] Ti A et al 2007 Int. J. Infrared Milli Waves 28243

[30] Asif M et al 2005 Phys. Lett. A 342175

[31] Asif M 2007 Phys. Plasmas 14062504

[32] K. Toi et al 1989 Phys. Rev. Lett. 62430

[33] Lichtenberg A J et al 1992 Nucl. Fusion 32495

[34] Itoh K et al 1992 Nucl. Fusion 321851

[35] Abdullaev S S 2002 J. Phys. A : Math. Gen. 352811

[36] Abdullaev S S 1999 J. Phys. A : Math. Gen. 322745 
[37] Igochine V et al 2006 Nucl. Fusion 46741

[38] Dumbrajs O et al 2005 Phys. Plasmas 12110704

[39] Meskat J P et al 2001 Plasma Phys. Control. Fusion 431325

\section{Figure captions}

Figure 1. The setup of diagnostics involved in present experiment. The three soft X-ray (SX) arrays named as SXA, SXC and SXE. Each array has 46 chords covering the whole plasma. The two chords in SXC, almost across the locations of two ECE channels, are prominently drawn. The hydrogen alpha-ray radiation $\left(H_{a}\right)$ nearly passes throng the plasma center. Figure 2. Time slice of the typical shot 110225. The waveforms are: plasma current, line-averaged density, $H_{a}$, 2-channels ECE, 2-channels SX and Mirnov signals from the top down. The bursts of $H_{a}$, ECE, SX radiations at $\mathrm{r}=-15.8 \mathrm{~cm}$ and the Mirnov signal oscillations are the main behaviors of relaxation. The relaxation makes the plasma current dropt $\sim 3 \%$.

Figure 3. Operational region of HT-7 tokamak: normal discharges (open circle), with complex modes (solid circle) and the previous disruptions (quadrangle surface denoted by 'D'). The solid line is a linear fit to the data denoted by solid circles.

Figure 4. Time slice of the Mirnov signals during a relaxation (a): raw signals and (b) the time-frequency spectrum. The arrows in (b) indicate 
the modes and their frequencies. The mode numbers at $\mathrm{f}=30-50 \mathrm{kHz}$ can not be indentified from Mirnov signals, and are defined as 'high (m, n) modes'.

Figure 5. The auto power spectrum density of the raw Mirnov signals. The arrows indicate three coherent peaks, and the broad spectrum is corresponding to the 'high $(\mathrm{m}, \mathrm{n})$ modes'.

Figure 6. The raw Mirnov signals at (a) and the three extracted signals by FFT method at the frequencies of $f(\mathrm{~b}), \sim 2 f(\mathrm{c})$ and $\sim 3 f(\mathrm{~d})$. The dynamics of the three low-order modes could be observed. The mode $\mathrm{m} / \mathrm{n}=2 / 1$ saturated before the mode $\mathrm{m} / \mathrm{n}=3 / 2$ is destabilized. The mode $\mathrm{m} / \mathrm{n}=5 / 3$ is also eventually destabilized. The SX signals at edge and center channels are shown in (e), as well as their enlargements in (f). The arrows with letters denote the time points for analysis: $\mathrm{A}(\mathrm{t}=437.5 \mathrm{~ms})$, $\mathrm{B}(\mathrm{t}=438.8 \mathrm{~ms}), \quad \mathrm{C}(\mathrm{t}=439.5 \mathrm{~ms}), \quad \mathrm{D}(\mathrm{t}=439.86 \mathrm{~ms}), \quad \mathrm{E}(\mathrm{t}=439.9 \mathrm{~ms}) \quad$ and $\mathrm{F}(\mathrm{t}=440 \mathrm{~ms})$. The time points $\mathrm{E}$ and $\mathrm{F}$ are not shown in Mirnov signals because of space limit.

Figure 7. Tomography results of SX perturbations at: $t=437.5 \mathrm{~ms}$ (a), $\mathrm{t}=438.8 \mathrm{~ms} \quad(\mathrm{~b}), \mathrm{t}=439.5 \mathrm{~ms} \quad(\mathrm{c}), \mathrm{t}=439.86 \mathrm{~ms} \quad(\mathrm{~d}), \mathrm{t}=439.9 \mathrm{~ms} \quad(\mathrm{e})$ and $\mathrm{t}=440 \mathrm{~ms}$ ( $\mathrm{f})$. The dash line circle is the estimated location of $\mathrm{q}=2$ rational surface in (a). The arrow indicates the weaker structure in (b) and (c) than that in (a) due to the destabilization of $m / n=3 / 2$ mode. Compared with the tomography contour in (d), the contour in (e) shows a shrink hot core due 
to the stochastic relaxation. In (f), the double arrow indicates a coherent structure of possessing the $\mathrm{m} / \mathrm{n}=2 / 1$ island properties.

Figure 8. The parameterized q-profile as a function of the normalized radius $\mathrm{r} / \mathrm{a}$, with consideration of the estimated location of $\mathrm{q}=2$ surface as well as the edge value.

Figure 9. The parameterized perturbation fluxes as a function of the normalized radius $\mathrm{r} / \mathrm{a}$. The free parameters $\alpha, \beta$ and $\gamma$ are fixed for each mode at different time points, and the marked perturbation values at edge are extracted from Mirnov signals at $\mathrm{t}=439.86 \mathrm{~ms}$.

Figure 10. The Poincaré mapping at $\mathrm{t}=439.86 \mathrm{~ms}$ where a stochastic annular belt of magnetic field lines could be observed. The perturbation fluxes are shown in figure 9.

Figure 11. The Poincaré mapping: at $\mathrm{t}=437.5 \mathrm{~ms}$ with $H_{21}^{\mathrm{exp}}=4 \times 10^{-4}(\mathrm{a})$, at $\mathrm{t}=438.8 \mathrm{~ms}$ with $H_{21}^{\text {exp }}=4 \times 10^{-4}$ and $H_{32}^{\exp }=8 \times 10^{-6}(\mathrm{~b})$, at $\mathrm{t}=439.5 \mathrm{~ms}$ with $H_{21}^{\text {exp }}=4 \times 10^{-4}, H_{32}^{\text {exp }}=1 \times 10^{-5}(\mathrm{c})$, as well as at $\mathrm{t}=440 \mathrm{~ms}$ with $H_{21}^{\text {exp }}=7.2 \times 10^{-4}$, $H_{32}^{\text {exp }}=8 \times 10^{-5}$ and $H_{53}^{\text {exp }}=6 \times 10^{-6}(\mathrm{~d})$. The arrows in (b) and (c) indicate the mode $\mathrm{m} / \mathrm{n}=3 / 2$ destabilized. The double arrow in (d) indicates the coherent structure of possessing the characteristics of $m / n=2 / 1$ island. 
Figures

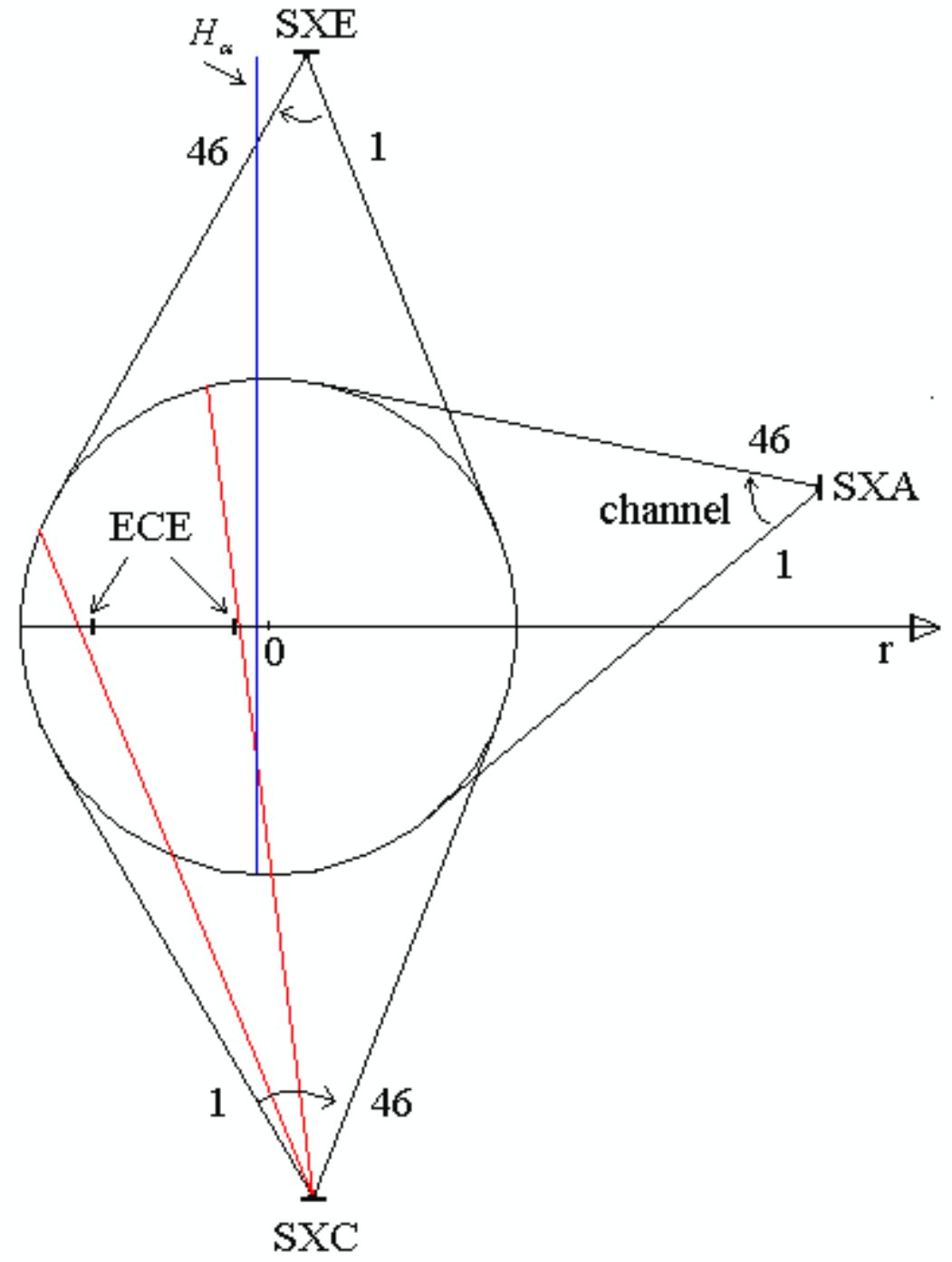

Figure 1 


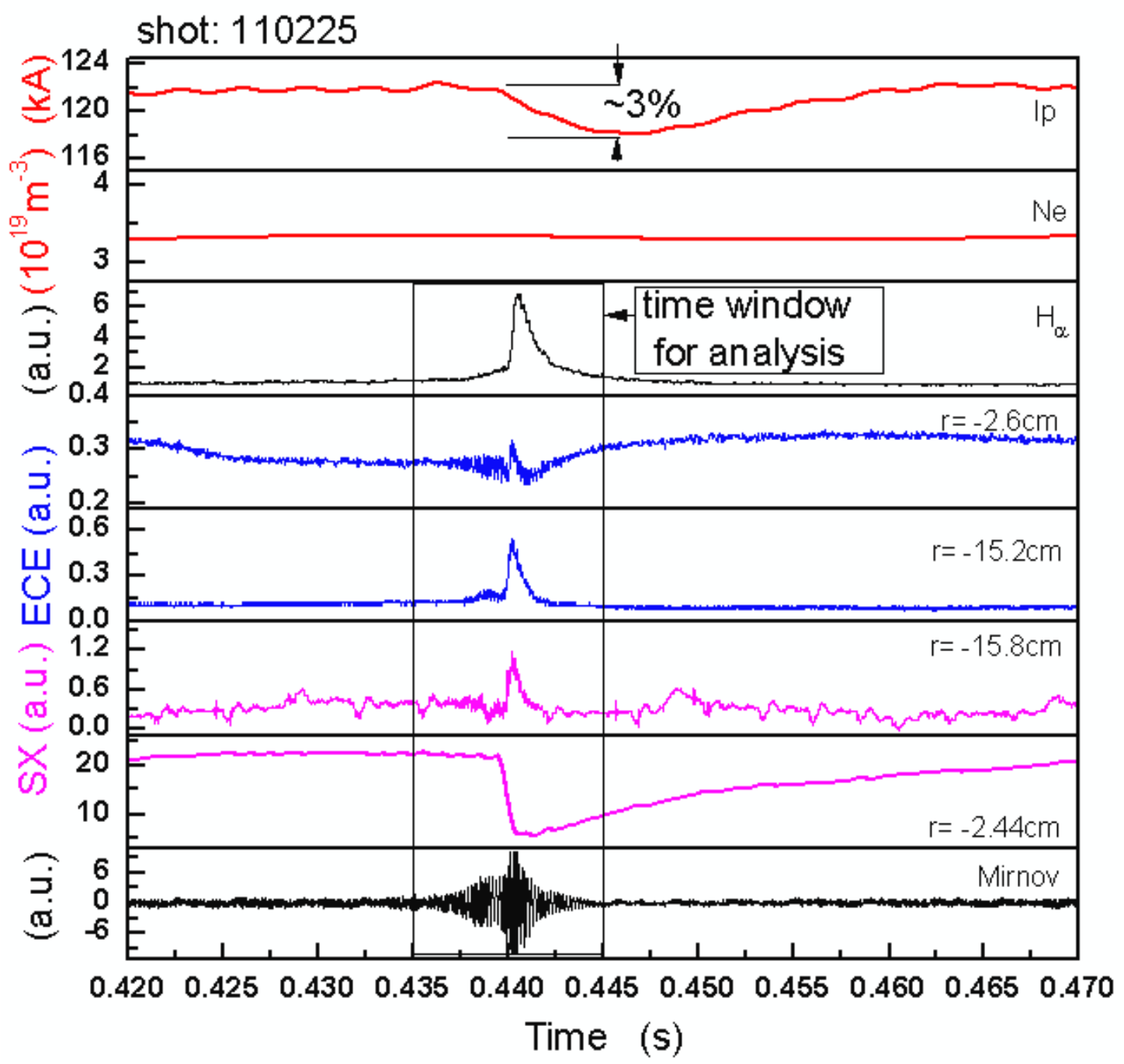

Figure 2 


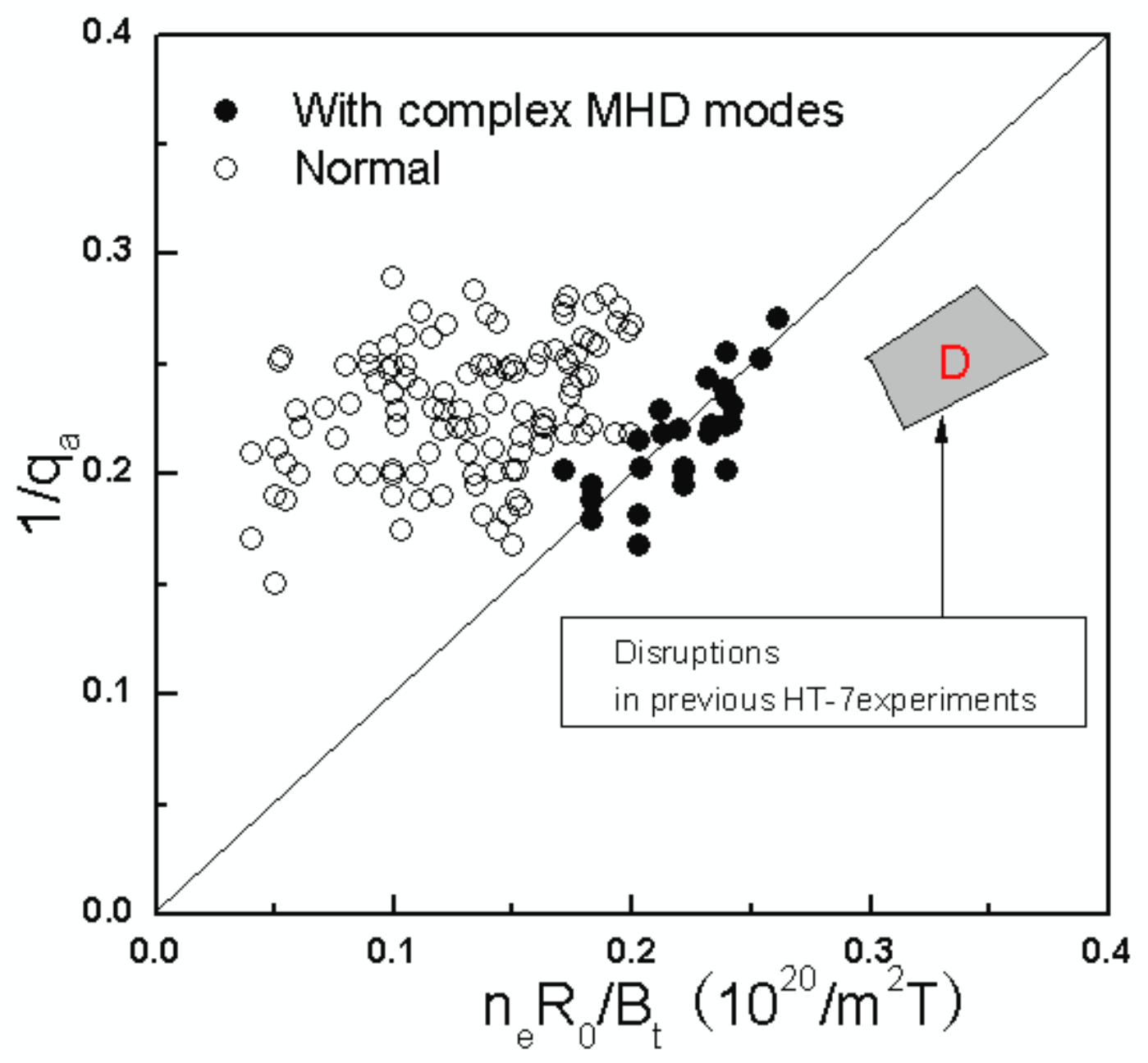

Figure 3 

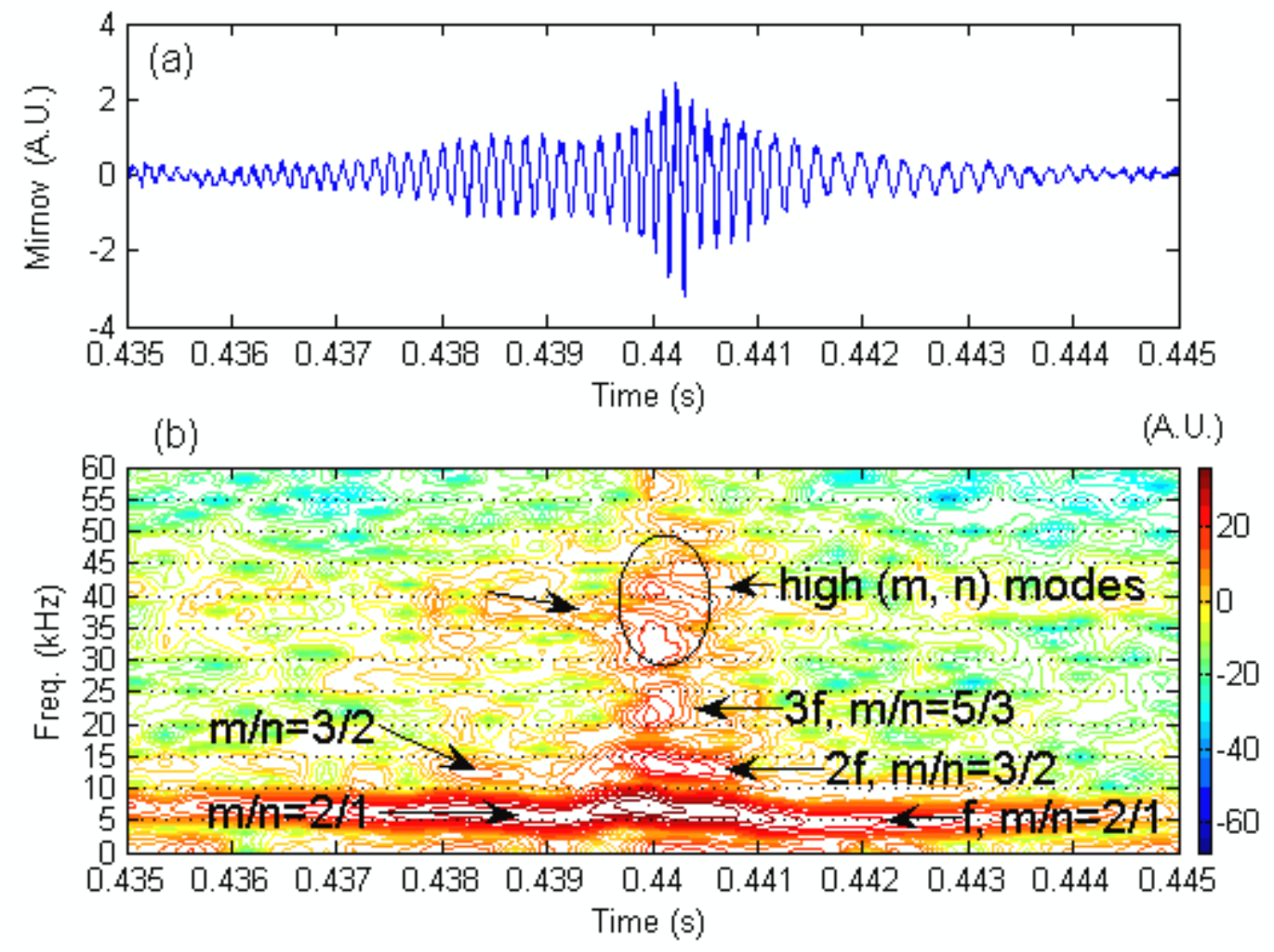

Figure 4

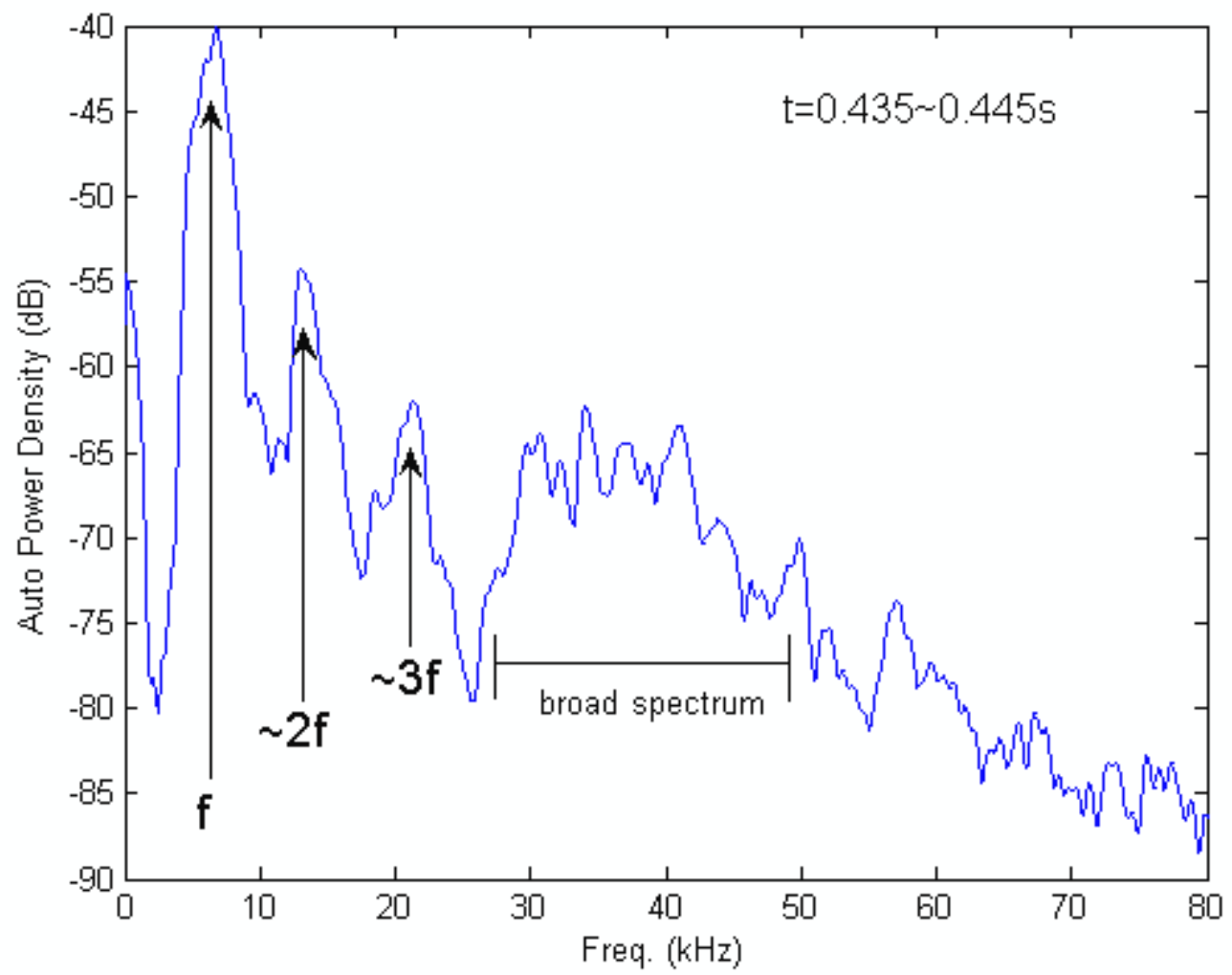

Figure 5 

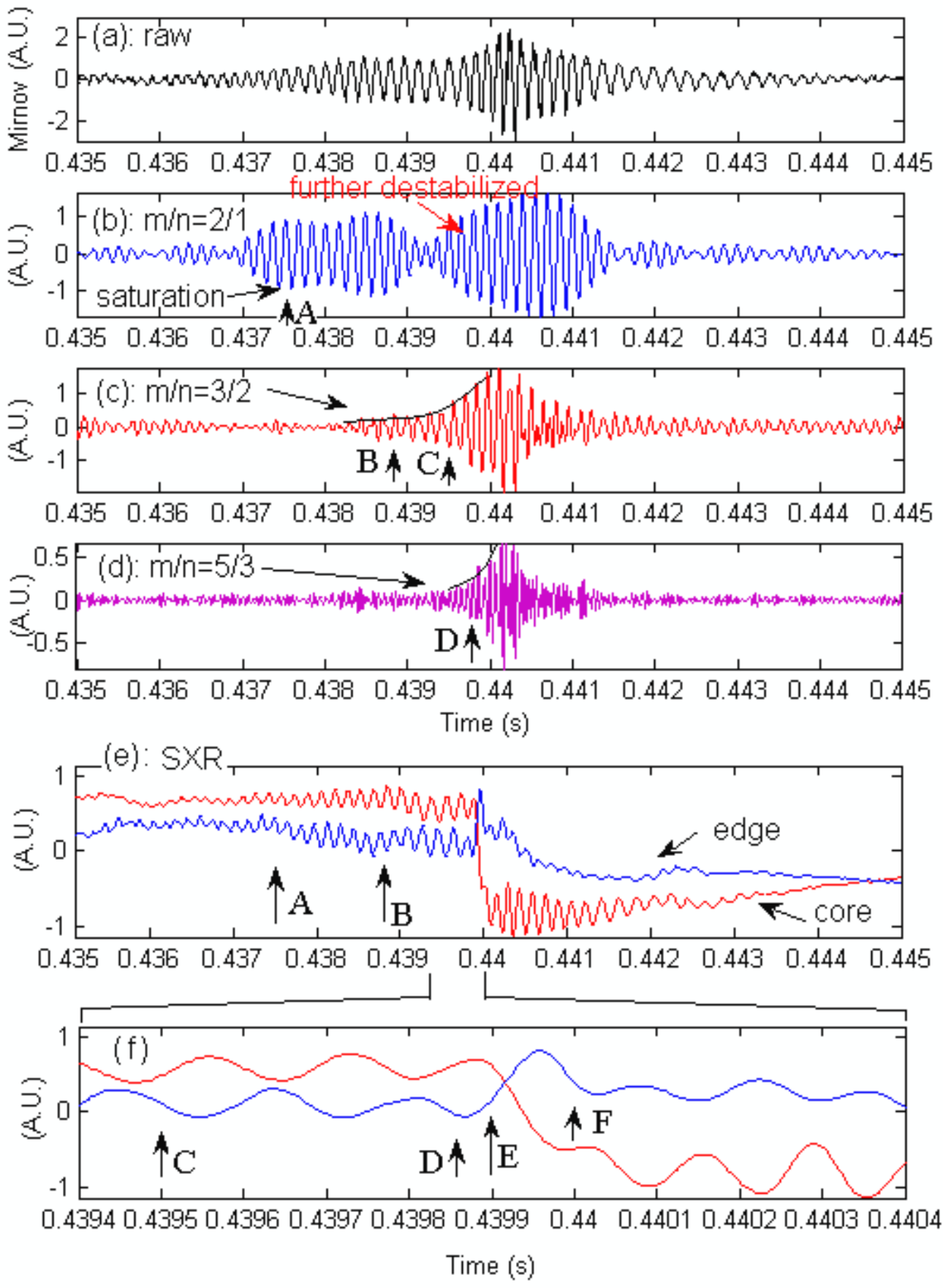

Figure 6 

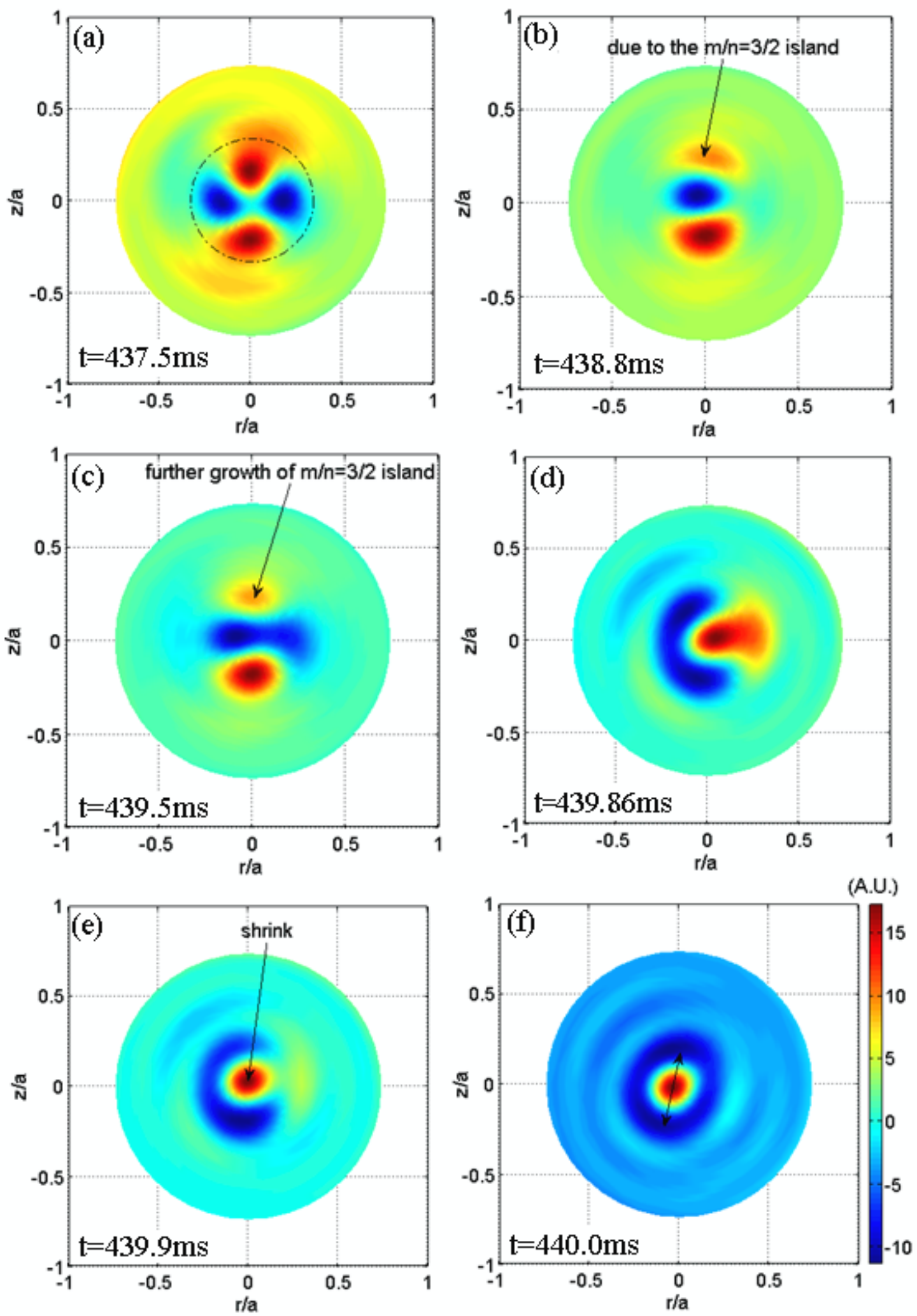

Figure 7 


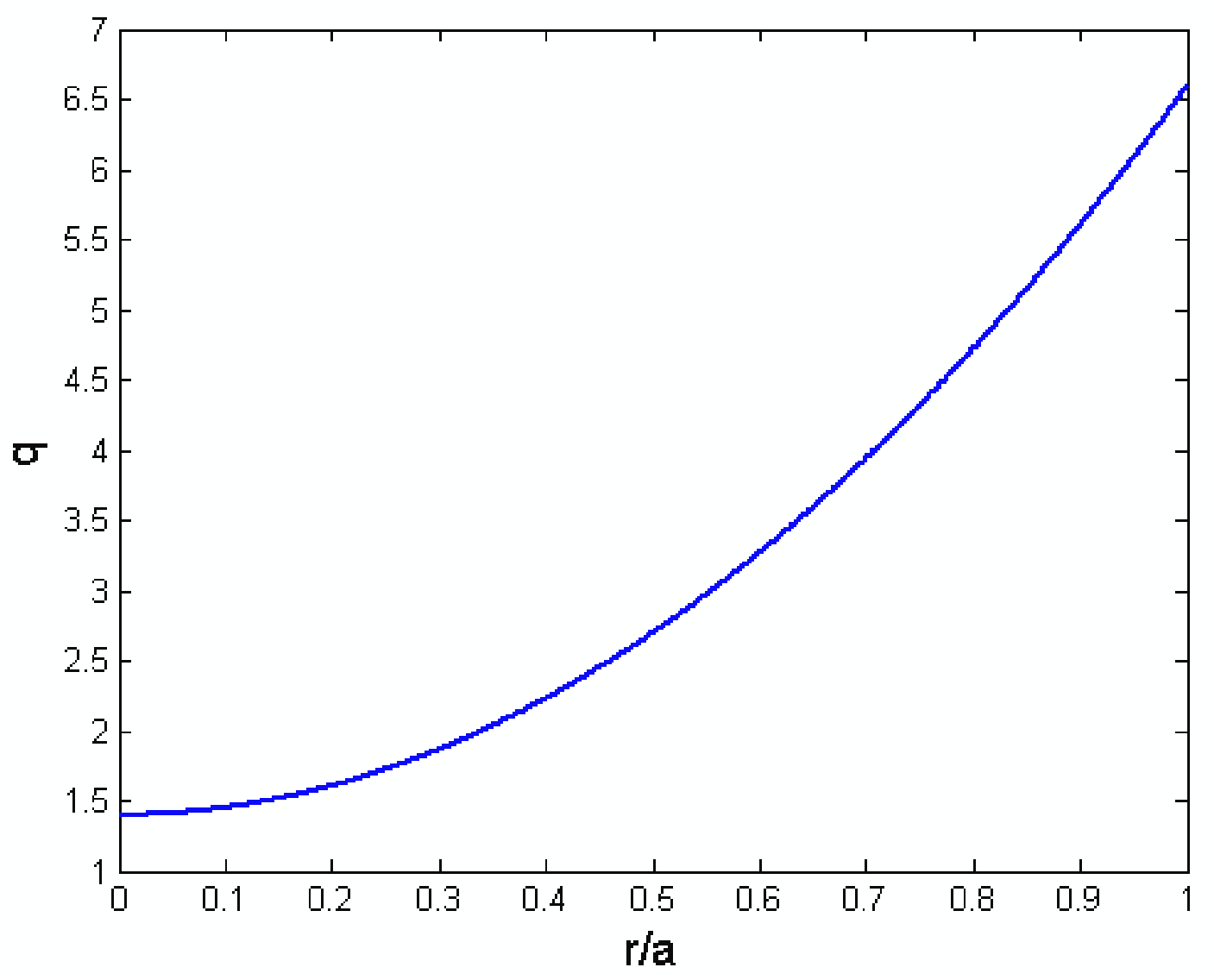

Figure 8 


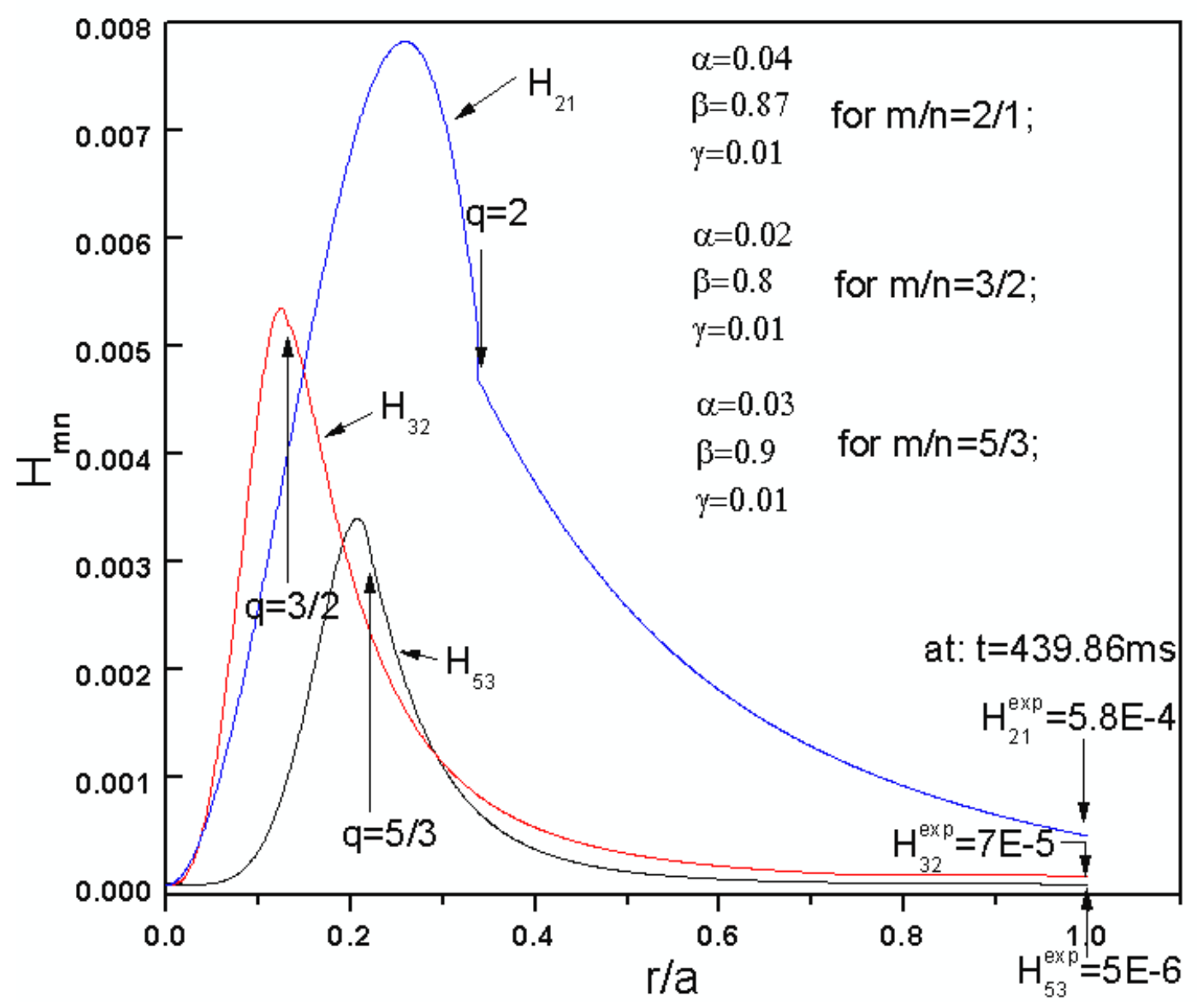

Figure 9 


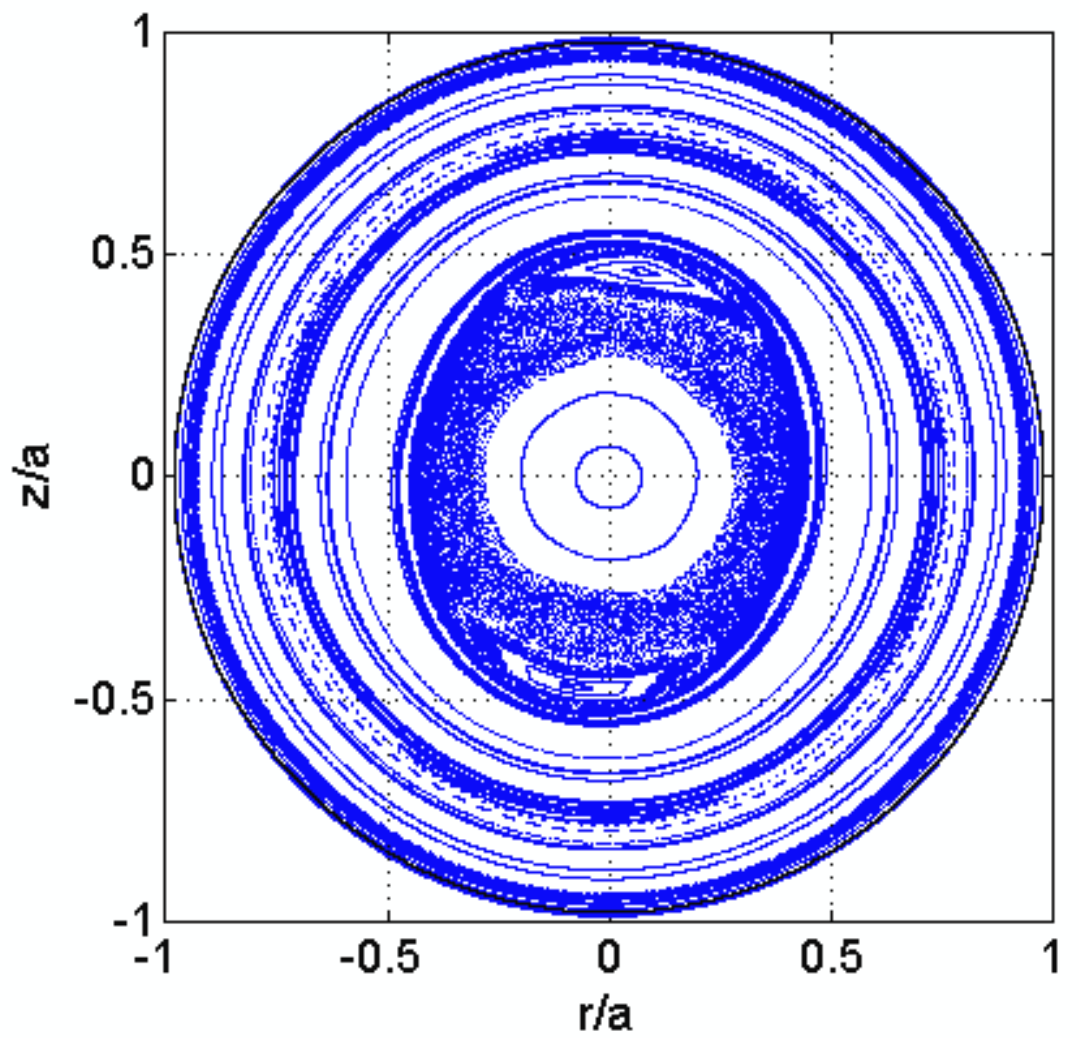

Figure 10 

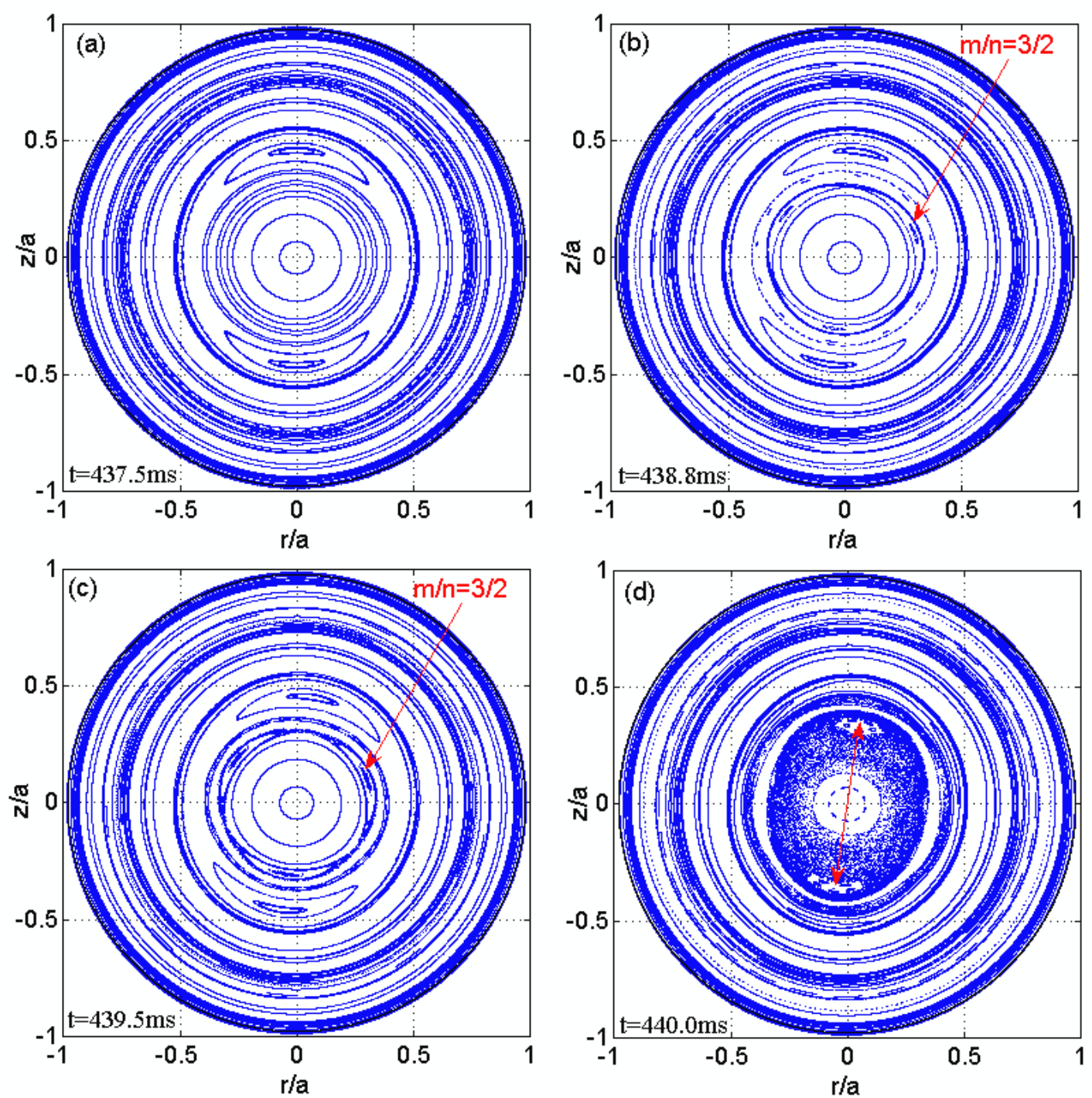

Figure 11 